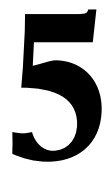

\title{
IDEA MODERNA DE UNIVERSIDAD: DE LA TORRE DE MARFIL AL CAPITALISMO ACADÉMICO ${ }^{1}$
}

\section{(THE MODERN IDEA OF UNIVERSITY: FROM THE IVORY TOWER TOWARDS ACADEMIC CAPITALISM)}

José Joaquín Brunner

Julio Roberto Labraña Vargas

Universidad Diego Portales

Francisco Ganga

Universidad de Los Lagos

Emilio Rodríguez-Ponce

Universidad de Tarapacá

DOI: $10.5944 / e d u c X X 1.22480$

Cómo referenciar este artículo/How to reference this article:

Brunner, J. J.; Labraña, J.; Ganga, F. y Rodríguez-Ponce, E. (2019). Idea moderna de Universidad: de la torre de marfil al capitalismo académico. Educación XX1, 22(2), 119-140, doi: 10.5944/educXX1.22480

Brunner, J. J.; Labraña, J.; Ganga, F. \& Rodríguez-Ponce, E. (2019). The modern idea of University: from the ivory tower towards academic capitalism. Educación XX1, 22(2), 119-140, doi: $10.5944 /$ educXX1.22480

\section{RESUMEN}

El presente artículo analiza la evolución de los discursos acerca de la universidad moderna a partir de la revisión reflexiva de textos clásicos y actuales sobre el tránsito desde la universidad como torre de marfil a la universidad emprendedora, examinando las tensiones que esta evolución genera en términos de principios formativos y organización del conocimiento. Para ello, este ensayo comienza argumentando que la universidad se hace parte de la Ilustración y accede a la modernidad cuando la Facultad de filosofía deja de ser conceptualizada como sierva de la Facultad de Teología y cuando deja, por lo tanto, de llevar su cola (philosophia theologiae ancilla), según ironiza Kant, para pasar en cambio a comprenderse como responsable de iluminar con una antorcha su camino. En las décadas siguientes el nuevo 
rol que asume la filosofía en la división del trabajo académico transforma las relaciones de la institución universitaria con el poder político y el poder religioso, abriendo las puertas, al mismo tiempo, hacia el Estado nacional y, más adelante, hacia la subordinación de sus actividades al capitalismo global. Consecuencia de lo anterior, los referentes normativos tradicionales de la universidad propiciados por Kant y Humboldt como responsable de la evolución de la sociedad moderna pierden plausibilidad y son sometidos a la crítica de las ideologías. El artículo concluye examinando futuras tendencias de la educación e investigación realizadas en las universidades contemporáneas y argumenta que la crisis de la idea moderna de la universidad es, de hecho, expresión de su carácter moderno que ahora se vuelve contra la misma institución.

\section{PALABRAS CLAVE}

Universidad; conocimiento; finalidad de la educación; facultad universitaria; Estado; capitalismo.

\section{ABSTRACT}

This article analyzes the evolution of discourses about the modern university by presenting a reflexive reading of classical and contemporary texts on the passage from a university conceived as an ivory tower to the entrepreneurial university, and the strains it creates in terms of formative principles and knowledge organization. To do so, the article begins by arguing that the university becomes part of the Enlightenment and accesses to modernity when the Faculty of Philosophy ceases to be conceptualized as the handmaiden of the Faculty of Theology (philosophia theologiae ancilla) and grows to be understood as the mistress' torchbearer, as ironically remarked by Kant. In the following decades, the new role that philosophy adopts in the division of academic labor changes the relationship of the institution with both the political and religious powers, opening the door on the road to the nation state and, further down, to the subordination of its activities to global academic capitalism. Consequently, traditional normative referents, as those proclaimed by Kant and Humboldt where universities were conceived as responsible for the evolution of modern society, lose plausibility and are subjected to the criticism of ideologies. The article concludes by examining future trends in education and research conducted in contemporary universities and by arguing that the crisis of the modern idea of the university is, in fact, an expression of its modern character that now turns against this very institution. 


\section{KEYWORDS}

University; knowledge; aims of education; university faculty; state; capitalism.

\section{DE SIRVIENTA A MAESTRA}

El comienzo de esta sociología histórica se halla en El Conflicto de las Facultades (Kant, 1798/2004) y se expresa en la creación de la Universidad de Berlín, en 1810. En aquel espacio entre la idea y la organización, su concepción y su práctica, se instala un orden autónomo, crítico y reflexivo para la razón pública que sustrae a la universidad del control religioso bajo el cual había nacido y del control imperial en el que se había desarrollado (Röhrs, 1996, pp. 16-32).

Clave en ese paso hacia un nuevo orden del conocimiento es el papel que juegan las diferentes facultades universitarias, denominación empleada desde el siglo XII para nombrar a las estructuras que organizan la transmisión de los saberes (Verger, 1992, pp. 41-45). Según el uso corriente de la época (Gieysztor, 1992, pp. 109-113), Kant divide a las Facultades en superiores - teología, derecho y medicina, formadoras de profesionales y reguladas por el gobierno- y una inferior, la facultad de Filosofía ${ }^{2}$, de la cual se espera que

independiente de las órdenes del gobierno en lo que se refiera a sus doctrinas, tenga la libertad, si no de dar órdenes, al menos de juzgar a todos los que se interesan por la ciencia, es decir, por la verdad, y en que la razón tenga el derecho de hablar con franqueza; porque sin esta libertad la verdad no podría manifestarse (lo que va en perjuicio del gobierno mismo). (Kant, 1798/2004, p. 23)

La filosofía, a la cual Kant sitúa en el más alto sitial, comprende en su época la física, la ética y la metafísica e incluye además los saberes posteriormente conocidos como humanidades y ciencias sociales (Howard, 2006, pp. 121-129).

La idea kantiana de universidad pone en curso de colisión a Dios y la razón, el antiguo orden cristiano y la naciente modernidad secular, la iglesia y el Estado, la teología y la filosofía. Fue el momento de la Ilustración, de pensar cada uno por sí mismo, por cuenta propia (Kant, 1784/2013). La universidad (moderna), tal era el postulado de Kant, debía instituir un lugar para el ejercicio público de la razón autónoma, con la función de controlar y servir a las facultades profesionales o «superiores» (Bermejo Castrillo, 2008; 
Brandt, 2008). Ello pues «todo depende de la verdad (condición primera y esencial de la ciencia en general); mientras que la utilidad que las facultades superiores prometen al gobierno solo tiene un valor de segundo orden» (Kant, 1798/2004, p. 35). Precisamente en este punto de su argumentación, el filósofo de la Ilustración introduce su famosa, e irónica, metáfora sobre la universidad al enunciar:

Sin duda, se podría conceder tal vez a la Facultad de teología la altiva pretensión de tomar a la Facultad de filosofía por sierva (pero siempre subsistiría el problema si esta precede con la antorcha a su graciosísima dama o si le sigue llevándole la cola); basta que no la despidan o la condenen a silencio, pues justamente esta modestia de la Facultad de filosofía, que solo quiere ser libre, que se la deje en libertad para encontrar la verdad en provecho de todas las ciencias y para ponerla a la libre disposición de las facultades superiores, esta modestia debe hacerla recomendable al gobierno mismo, como indispensable y ponerla al abrigo de toda sospecha. (Kant, 1798/2004, pp. 35-36)

Sobre estas bases, la cultura crítica emanada de la Ilustración es acogida en la Facultad de filosofía, apoyada además por las nacientes ciencias físicas y naturales surgidas durante el siglo XVII, mientras la cultura orientada a la práctica, orientada utilitariamente, quedaría en manos de las facultades profesionales, particularmente leyes y medicina.

\section{DE BERLÍN AL MUNDO GLOBAL}

Desde el punto de vista institucional, la idea kantiana de una universidad sede de la razón autónoma ejercida y enseñada-incluso conflictivamente-por la Facultad de filosofía, chocaba directamente con la realidad universitaria germana de mediados del siglo XVIII. Según escribe un historiador,

las universidades alemanas se encontraban mal aspectadas cuando amanecía el siglo de las luces, repletas de problemas internos, entregada a la ciencia aristotélica, comprometida con una pedagogía pasada de moda y frecuentemente poco receptiva o incluso de espaldas al rico momento de fermento intelectual asociado a los nombres de René Descartes, Francis Bacon, Sir Isaac Newton, Voltaire y Diderot. (Howard, 2006, p. 80)

Ellas enfrentaban además una extendida crisis: aguda disminución de la matrícula, proliferación de pequeñas instituciones dispersas, caída del ingreso público, profesores que debían complementar sus ingresos con trabajos externos, nepotismo académico y estudiantes caracterizados por 
una subcultura disoluta y violenta (Schwerhoff, 2013). Varios miembros de la intelligentsia, influidos por la Ilustración y la revolución científica, prefirieron contratarse independientemente antes que incorporarse a alguna institución: Leibniz es el caso más famoso. Pero también figuras del reino de las letras como Goethe, Mendelssohn, Klopstock y Lessing criticaron fuertemente a las universidades de su tiempo (Howard, 2006, pp. 84-84).

En el resto de Europa, la universidad experimenta sucesivas oleadas de reforma durante los siglos XVII al XIX, producto de su expansión, su diferenciación entre diversos tipos de instituciones y su creciente carácter profesionalizante (Frijhoff, 2003a) impulsado por los Estados, los que por primera vez buscan ajustar las funciones de la universidad a los requerimientos del mundo del trabajo. Según señala Frijhoff,

esta metamorfosis de las universidades, de ser instituciones que producían más o menos lo que les complacía a ser organizaciones cuya producción era guiada por las necesidades y demandas del mercado, es quizá la característica más llamativa de este período. Ciertamente es aquella que más fuertemente ha afectado el desarrollo de las universidades hasta el presente. (Frijhoff, 2003b, p. 80)

La creación de la Universidad de Berlín, inspirada en la filosofía de Kant e impulsada por el neohumanismo alemán de Wilhelm von Humboldt y su grupo de esclarecidos intelectuales (el más importante entre ellos, y su principal colaborador, el pastor protestante, escritor y teólogo Friedrich Schleiermacher), se produce por tanto en un contexto de decadencia de la universidad y en una Europa agitada por las transformaciones de su educación superior. Como señala Howard (2006), ella constituye una reacción frente a la percepción de decadencia intelectual.

Simbólicamente ella es considerada la primera universidad moderna en cuanto a su relación con el conocimiento y su propósito de encarnar la razón autónoma. Como mencionamos, pone a la Facultad de Filosofía en el lugar prominente, otorgándole el rol más influyente; la antorcha que ilumina el camino. Con el tiempo, la universidad humboldtiana se convertirá en el paradigma institucional del mundo universitario a nivel global, bajo la forma de la research university, el hogar de las ciencias a gran escala de la segunda mitad del siglo XX y primera parte del siglo XXI, aunque en muchos casos se tratase de una interpretación mitológica (Ash, 2006, 2008). ${ }^{3}$

El historiador noruego Thorsten Nybom (2007) sostiene que el establecimiento de este nuevo tipo de universidad en la capital prusiana respondió a varios factores. De carácter ideológico, primero, por la combinación entre neohumanismo (clasicismo), Ilustración germana 
tardía e idealismo pre-romántico alemán, cuyo punto de partida sería el texto de Kant sobre el conflicto de las facultades (McClelland, 2008, pp. 111-149). Factores políticos, enseguida, el más importante de los cuales habría sido la derrota de Prusia en manos de los ejércitos napoleónicos y el consiguiente esfuerzo de reconstrucción del Estado presidido por el gabinete reformista del Canciller Hardenberg y su ministro Von y Zum Stein, del cual Wilhelm von Humboldt fue por un breve, pero productivo tiempo, secretario de educación y cabeza de la reforma de los sistemas escolar y superior (Clark, 2006, pp. 312-344). Factores de mentalidad o cultura, a continuación, por la afortunada concentración de inteligencia creativa, seguramente única, como sugiere Nybom (2007), que generó una verdadera obsesión de la élite prusiana por la educación en general (Ringer, 1990, pp. 15-17). Por último, factores de carácter institucional, relacionados con el decaimiento del entorno interno y externo del sistema universitario germano, según exploramos más arriba. Recuérdese el dicho atribuido al rey Federico Guillermo III de Prusia, quien, tras la derrota frente a los ejércitos napoleónicos, habría declarado: «el Estado tiene que reemplazar con poder intelectual, el poder físico que ha perdido» (Barkhoff, 2017, p. 38).

\section{DE LA BILDUNG HUMANISTA AL KULTURSTAAT}

Entonces, ¿cuál fue la combinación de ideas e ideales que Humboldt reunió y propuso como base de la nueva universidad alemana?

El mismo Nybom (2007), en una perspectiva convergente con otros especialistas (Horlacher, 2014; Abellán, 2008), describe los elementos centrales de esta filosofía de la siguiente forma: conocimiento como una entidad única e indivisible; unidad de investigación y enseñanza; primacía de la ciencia (Wissenschaft) ${ }^{4}$ y la investigación; presupuesto para un nuevo orden institucional y jerarquía cognitiva; búsqueda libre de la verdad, sin apremios externos, por tanto en relativo aislamiento o retraimiento y con independencia (Einsamkeit und Freiheit); libertades de enseñanza y estudio (Lehr und Lernfreiheit); y creación de una cultura nacional unificada con las ciencias y la universidad como centro. Formulado brevemente, esta filosofía considera la formación e investigación realizada en las universidades alemanas como un segundo imperativo categórico, junto a la defensa nacional, base de un moderno Kulturstaat (Estado de cultura o Estado cultural) (Howard, 2006, pp. 212-266).

Estas ideas se contraponen al vocacionalismo profesional de las antiguas facultades superiores y a la fragmentación de las ciencias emergentes durante el siglo XVIII. En contraste, el centro de la visión humboldtiana es la idea de Bildung (Hamann, 2015) y no —como suele 
postularse- la combinación de las funciones académicas de docencia e investigación. Por el contrario, dicha combinación es relevante solo en tanto ofrece oportunidades institucionales a estudiantes y académicos de avanzar en su formación, que es fin declarado de la universidad (Hastedt, 2012). Bildung es una filosofía formativa; una concepción del desarrollo y la transformación basada en el autocultivo armónico de las facultades humanas, a la manera que Goethe lo postulase en su novela Wilhelm Meister (Benziman, 2018).

De hecho, Bildung es un término difícil de traducir (Horlacher, 2014) que, además, ha experimentado profundos cambios a lo largo de los últimos dos siglos (Hamann, 2011). Refiere a una idea educativa, consistente en la participación del sujeto en su propia formación. Es algo distinto a la enseñanza e instrucción. En el vocabulario del neohumanismo remite a la idea griega de Paideia (Jaeger, 1980) la educación del hombre libre que se dirige hacia un ideal, un modelo ambicioso de persona virtuosa. Sin embargo, a diferencia del pensamiento griego, el concepto de Bildung asigna especial importancia a la capacidad de desarrollar las cualidades de cada individuo siguiendo sus propios intereses y motivaciones; representa, en este sentido, la expresión de lo universal en cada individuo.

En la tradición socrática equivale a vivir una vida examinada; en el lenguaje actual, una vida con sentido. Es extraer algo desde el fondo —el alma humana, su potencialidad- más que enseñar desde arriba, para inculcar un conjunto de verdades o una forma de ser adaptada a las demandas externas provenientes del Estado o la iglesia. En concepto de Humboldt es, por consiguiente, una educación general —liberal, se dice hasta hoy - desprovista de cualquier consideración de utilidad o usabilidad, de empleabilidad diríase ahora, no porque ella sea irrelevante, sino porque está asegurada por el desarrollo armónico de las potencialidades de cada persona. Es una educación comprensiva y en profundidad, que obedece al propósito humboldtiano del máximo desenvolvimiento de las habilidades y destrezas hasta llegar a conformar un todo armónico (Zovko \& Dillon, 2018).

En un escrito póstumo, al que se ha dado el nombre de Teoría de la Bildung, Wilhelm von Humboldt, escribe:

la tarea superior de la vida es llenar el concepto de «humanidad» —en nuestra persona, tanto durante la duración de nuestra existencia como más allá a través de las huellas que dejamos por nuestras actividadesdel contenido más rico posible; esto solo se logra asociándose al mundo mediante una interacción lo más comprensiva, vital y libre posible. (Humboldt, 1995 citado en Nordenbo, 2003, p. 32) 
En fin, una Bildung humboldtiana así entendida, núcleo de su idea de universidad, es un asunto individual y colectivo a la vez, público y privado, institucional y al mismo tiempo íntimamente personal; es, de esta manera, una pieza crucial de la realización del ideal del Kulturstaat germano a través de la reforma del sistema de educación superior (Ringer, 1990, pp. 113-127).

En este punto, la idea de Bildung —al relacionarse con el momento kantiano de la idea de universidad - da paso a una nueva definición del proceso de autoformación en términos de autonomía racional de las personas. No solo equivale a la emancipación de la razón respecto de cualquier tutelaje externo, sino que, además, abre paso al proyecto políticocultural de una progresiva racionalización de los mundos de vida, en cada una de las esferas de valor que posteriormente conformarán la sociedad moderna.

\section{DE ARTESANOS A EMPRESAS DE CONOCIMIENTO}

Llegamos aquí, un siglo más adelante-tiempo durante el cual la idea y la institución de la universidad experimentan cambios sustanciales (Rüegg, 2004) — a una nueva situación sociológica de esta organización, que Max Weber resume de la siguiente forma:

Ahora podemos ver con claridad que la evolución más reciente de nuestras universidades se mueve, en amplios campos de la ciencia, en la dirección de las universidades americanas. Los grandes institutos de Medicina y de Ciencias Naturales son empresas de «capitalismo de Estado».... Nuestra vida universitaria alemana se está americanizando en aspectos muy importantes, como nuestra vida en general, y estoy convencido de que esta evolución irá afectando también a aquellas especialidades donde, como ocurre en la mía en gran medida, el artesano mismo es propietario de sus medios de trabajo (básicamente, la biblioteca) de la misma manera que en el pasado el viejo artesano era el propietario en su oficio. Esta evolución se encuentra en plena marcha. Las ventajas técnicas de esta evolución son indudables, como en todas las empresas capitalistas y al mismo tiempo burocratizadas. Pero el «espíritu» que reina en ellas es distinto a la antigua atmósfera de las Universidades alemanas. (Weber, 1917/2008, p. 27)

Lo que Weber anuncia tempranamente allí es el inexorable advenimiento del capitalismo académico en alguna de sus variedades; más abierto a la coordinación político-administrativa proporcionada por el Estado, en algunos casos, o más propenso a una coordinación provista por los mercados en otros (Brunner, 2017; Jessop, 2017). Y, junto con aquel 
advenimiento, la secuela — también inevitable — de las contradicciones culturales del capitalismo académico; por ejemplo, del trabajo artesanal versus industrial, o entre organización burocrática y dominio de una oligarquía académica, o entre la atmósfera de una universidad racional a la Kant o una instrumentalmente racionalizada a la manera de Weber.

En el intertanto, ¿qué sucede-en las circunstancias de modernidad tardía o tiempos posmodernos-con la universidad y sus condiciones humboldtianas de posibilidad?

Dicho sucintamente: pasa de ser una institución—orientada por la idea de Bildung, y gobernada por sus propios académicos al amparo del Estado-a ser una organización académica preocupada de gestionar estratégicamente sus asuntos en un ambiente competitivo y de dar cuenta de sus resultados (Krücken \& Meier, 2006), al tiempo que los sistemas nacionales de educación superior se masifican, diferencian y burocratizan y giran su economía política hacia los mercados (Bleiklie et al., 2015).

La educación en sus diferentes niveles, especialmente el superior, se concibe ahora en términos de competencias claves para la economía. Digamos así: se busca paradojalmente crear una Bildung con fines ocupacionales, al servicio de la economía laboral (Zovko \& Dillon, 2018), dando lugar a su crítica romántica cuyos orígenes pueden ser rastreados hasta Nietzsche (Llinares, 2008). Concomitantemente surge una nueva jerarquía de Facultades y se redefinen los órdenes del conocimiento (Habermas, 1987). En el centro de la idea de universidad aparecen ahora la producción de capital humano y el conocimiento como capital (Becker, 1994), cuyo propósito es contribuir a la productividad del trabajo y la competitividad de las empresas y la nación.

El eje de la política universitaria se economiza, tanto en los países desarrollados que conciben el crecimiento como innovación schumpeteriana como en los países en desarrollo que aspiran a cerrar la brecha con los anteriores mediante la incorporación de tecnologías. En torno a esta visión economizante se organizan poderosas burocracias locales, nacionales e internacionales que proclaman a la educación-desde la cuna hasta la tumba-como una prioridad de los países y una herramienta para la movilidad social y la democracia política. Al frente aparecen los factores institucionales de competencia, la comparación y el benchmarking de los sistemas nacionales de educación superior y los rankings que determinan la marca reputacional de las universidades, fenómeno que a escala global alimenta una carrera por personal, recursos y prestigio para así calificar como world-class university. 
Cada vez más distante de sus antecedentes históricos y bases espirituales, la universidad se halla hoy envuelta por mercados, redes sociales y medios de comunicación, una industria trasnacional del conocimiento, políticas públicas que incesantemente producen regulaciones y establecen regímenes de evaluación de resultados, todo esto con un fuerte impacto en la gobernanza y la gestión de las universidades (Paradeise et al., 2009).

En este contexto se plantea la pregunta actual de qué ocurre cuando Schumpeter sale al encuentro de Humboldt (Pinheiro, 2015) y ambos buscan determinar el rumbo futuro de la universidad emprendedora (Etzkowitz, 2017). Pregunta que en América Latina puede reformularse en estos términos: ¿qué sucede con la «universidad militante» criticada por José Medina Echavarría (1967, p. 169) cuando en torno de ella resuenan los ecos de Humboldt llegados a estas latitudes?

\section{DE LA TORRE DE MARFIL A LAS ANARQUÍAS ORGANIZADAS}

En vez de la unidad indivisible del conocimiento, tenemos hoy una profunda división ya no solo entre las culturas de las ciencias y las humanidades (Snow, 2012), sino la subdivisión de ambas en un número creciente de tribus y subtribus disciplinarias. El mundo del conocimiento se halla en constante expansión, descomponiéndose en especialidades y temas que se conectan entre sí a través de redes y fenómenos de serendipity (Habermas, 1987). Las Facultades de hoy son como islas dentro de archipiélagos que continuamente buscan ajustarse a una ecología cambiante y al cambio climático dentro de las organizaciones académicas. Contra las expectativas de Kant, la Facultad de Filosofía, en los términos retratados por él, apenas logró establecerse en unas pocas partes como el sector hegemónico entre los campos del saber. En efecto, pronto debió desprenderse de las ciencias naturales y exactas y se separó en varias ramas especializadas del conocimiento, como Artes, Humanidades y Letras (Rothblatt, 2011, pp. 243-244) ${ }^{5}$. De este modo fue desplazada de su posición dominante por la renovada fuerza de las antiguas Facultades profesionales, particularmente aquellas encargadas de la investigación y enseñanza de Tecnologías y Medicina.

La unidad entre docencia e investigación, concebida por la generación de Humboldt cual motor de la universidad moderna, mantiene su atractivo como ideal retórico pero, en la práctica, ha desaparecido de la mayoría de programas de pregrado y cursos de maestría (Ash, 2006, 2008), para refugiarse casi exclusivamente en los programas de doctorado. La racionalización de la enseñanza académica masiva no ocurre ya en torno al eje de la unidad entre docencia e investigación sino que se realiza con acuerdo a una metáfora industrial: mediante la producción estandarizada 
de cursos, con formas más y más tecnologizadas de provisión, como puede observarse, por ejemplo, en los massive open online courses.

La primacía de las ciencias, la erudición y la investigación que Humboldt propuso como desiderátum funcional de la universidad alemana, y que luego se materializaría en las research universities de los EE.UU., Gran Bretaña y el resto del mundo (Röhrs, 1996), es hoy la marca característica de un pequeño porcentaje de universidades, hallándose casi ausente de las demás universidades y de decenas de miles de instituciones no-universitarias de educación terciaria. Al compás de lo observado por Weber sobre la americanización de la universidad alemana hace ya un siglo, la propia investigación académica ha devenido big science, organizada industrialmente ${ }^{6}$ y concentrada en unas pocas universidades de clase mundial o regional en cada continente.

La idea de que la «verdad» ha de buscarse en relativo aislamiento y con plena libertad (Einsamkeit und Freiheit), debiendo la universidad ofreceren sentido positivo-algo semejante a una torre de marfil (Shapin, 2012), está en las antípodas de la realidad universitaria posmoderna. La universidad se ha transformado en una anarquía organizada, ruidosa, dinámica, con fuertes rasgos mercantiles y empresariales, sensible a las demandas de su entorno, en constante tensión con la sociedad civil y el Estado, preocupada de generar ingresos e invertir en variados proyectos, con sus claustros inundados de música y sus muros de graffiti, donde las tribus académicas coexisten interiormente ensimismadas pero conectadas hacia el exterior por múltiples canales y contratos, intercambios y flujos de información, recursos, influencia y prestigio. Este es el momento del pleno despliegue del capitalismo académico (Cantwell \& Kauppinen, 2014; Slaughter, 2014) en sus diversas variedades en cada territorio (Jessop, 2017).

Si bien las libertades clásicas de la vida académica-de enseñar y estudiar, aprender e investigar-se hallan consagradas en los estatutos universitarios y las leyes de la mayoría de los países, se ejercen un ambiente y culturas organizacionales dominadas por el productivismo académico, la burocratización y una incesante evaluación del desempeño individual y colectivo que la profesión académica percibe como una fuente de alienación. Siguiendo el Fausto de Goethe (1832/1981), la máxima de la academia moderna parece ser: "A quien siempre con diligencia trabaja podemos redimido $»^{7}$ (p. 359).

En estas circunstancias, la Bildung ofrecida por la época posmoderna se vuelve-por así decirlo-su contrario; desaparece la noción del hombre autocultivado que desarrolla al máximo su humanidad y, en adelante, se plantea la lucha entre la formación de especialistas o expertos y la creación 
de seres humanos cultos. Similares debates aparecen en otros países (Readings, 1996; Hütter, 2013). Según anota el mismo Weber con relación a este tema,

tras todas las discusiones del presente en torno a los fundamentos de la cultura se encuentra en algún punto decisivo el combate del tipo del «especialista» contra el antiguo "hombre culto», combate que en todas las más íntimas cuestiones culturales se halla determinado por la incesante propagación de la burocratización de todas las formas de poder públicas y privadas y por la importancia cada vez mayor del saber especializado. (Weber, 1922/2014, p. 1188)

\section{DE LA RAZÓN A LA RACIONALIZACIÓN}

Sin duda, el ideal humboldtiano de la Bildung (emparentado con el ideal del 'hombre culto', de Weber) (Ringer, 2004), igual que la idea kantiana de universidad, han sido reemplazados por el 'especialista' proveniente de las Facultades 'superiores', por los saberes provistos por las ciencias 'americanizadas' y por la universidad emprendedora (Clark, 1998) y performativa en el sentido de Lyotard (1984), conceptos ligados, respectivamente, a los dominios de la economía y la racionalización instrumental de la sociedad. La universidad desacoplada de los ideales de la Ilustración se vuelve ahora progresivamente un dispositivo decisivo para la burocracia estatal, la sociedad civil y los mercados, no por su valor cultural, sino por sus réditos económicos. De la universidad de la razón autónoma ha nacido la universidad racionalizada al máximo, la cual, a su vez, se ha convertido en un poderoso agente de la racionalización científico-técnica de la sociedad (Zovko \& Dillon, 2018).

Junto con Weber cabe preguntar entonces, ¿qué representa-desde el punto de vista práctico-esta racionalización operada a través de la ciencia y la técnica? Su respuesta es conocida: indica «que todas las cosas se pueden dominar mediante el cálculo. Esto significa, sin embargo, la desmagificación del mundo» (Weber, 1917/2008, p. 35) ${ }^{8}$. Este desencantamiento provocado por el avance de la racionalidad científico-técnica, ¿posee algún sentido que trascienda lo puramente práctico y técnico y permita ir más allá de un horizonte de control? Solo por un breve momento, dice Weber, el trabajo científico, indirectamente influenciado por el pietismo, pudo considerarse a sí mismo como un camino hacia Dios. Mas a continuación este autor se pregunta con incredulidad,

«¿Y hoy? ¿Quién cree hoy todavía... que los conocimientos de la astronomía o de la biología o de la física o de la química pueden 
JOSÉ JOAQUÍN BRUNNER, JULIO ROBERTO LABRAÑA VARGAS, FRANCISCO GANGA, EMILIO RODRÍGUEZ-PONCE

IDEA MODERNA DE UNIVERSIDAD: DE LA TORRE DE MARFIL AL CAPITALISMO ACADÉMICO

enseñarnos algo sobre el sentido del mundo o al menos algo sobre el camino que podría descubrir ese «sentido», si es que existe? Si enseñan algo, ¡esos conocimientos son adecuados para matar de raíz la fe en que existe algo así como un «sentido» del mundo!» (Weber, 1917/2008, p. 39)

En breve, esta racionalidad promueve un control instrumental del mundo y un desencantamiento de las ilusiones que originalmente se creyó acompañarían a la ciencia. En cambio, no puede ofrecer respuestas «a la única pregunta importante para nosotros, la de qué debemos hacer y cómo debemos vivir», según dice el sociólogo alemán citando a Tolstoi (Weber, 1917/2008, p. 39).

Dicho en otras palabras, cuestiones centrales del pensamiento acerca de la Bildung no pueden responderse hoy desde la Facultad de Ciencias, pues «la ciencia no es hoy un don de visionarios y profetas que distribuyen bendiciones y revelaciones, ni parte integrante de la meditación de sabios y filósofos sobre el sentido del mundo», según dice Weber (1917/2008, p. 42). Menos aún pueden responderse desde las Facultades profesionales, a menos que uno crea en soluciones técnicas para problemas existenciales; esto es, literalmente, el principio del value for money.

En cambio, ¿podrían las cátedras de la Facultad inferior-aquellas dedicadas a las humanidades, las artes y a una proporción de las ciencias humanas y sociales-ofrecer en la actualidad una respuesta si no salvífica a lo menos liberadora? Weber rechaza esta posibilidad: las profecías lanzadas desde la cátedra podrán crear sectas fanáticas, dijo una vez, pero nunca crearán una auténtica comunidad de valores y sentidos. Hablando a una audiencia de jóvenes, señala:

...si ese profeta no existe o si ya no se cree en su mensaje, es seguro que no conseguirán ustedes hacerlo bajar de nuevo a la tierra intentando que millares de profesores, como pequeños profetas pagados o privilegiados por el Estado, asuman en las aulas su función. (Weber, 1917/2008, p. 49)

En la universidad actual, científico-técnica, secular y emprendedora, el lugar de las ciencias del espíritu, incluida la filosofía, no es el de sirvienta pero tampoco porta la antorcha que ilumina el camino. En estas circunstancias, «tiempo sin profetas y ajeno a Dios» como lo llama Weber, ¿no podría justificarse un retorno a la más antigua de las Facultades, la de Teología, para llenar el vacío causado por el silencio de las ciencias ante la pregunta por el sentido? Weber, que siempre aspiró a mirar de frente la realidad, responde negativamente: 
La teología no puede darle fe ... a quien carece de ella. Tampoco puede dársela ninguna otra ciencia. Por el contrario, en toda teología "positiva» llega el creyente a un punto en el que adquiere validez la máxima agustiniana "credo non quod, sed quia absurdum est». (Weber, 1917/2008, p. 51) ${ }^{9}$

Si todavía hay un lugar para la teología en la universidad, ha dicho un teólogo católico con resignada ironía, ya que no puede estar sentada a la mesa junto a las demás ciencias duras seculares, «entonces al menos debe estar debajo de la mesa para alimentarse con las migajas que caen de esa pasmosa elaboración de conocimientos ... propia de la era posmoderna» (Hütter, 2013, p. 753).

En breve, la Bildung, que representó en su momento una respuesta positiva de la razón autónoma situada entre la religión y el Estado, una narrativa de la auto edificación humana en medio de la naciente modernidad ha perdido su fuerza. En el presente-cuando la universidad se ha vuelto performativa y la educación superior ha adquirido el carácter de un consumo masivo en medio del capitalismo académico-ella se torna francamente anacrónica.

Estamos de regreso a fojas cero. Las Facultades vuelven a sus querellas. E igual como los antiguos dioses, disputan entre sí sobre la comprensión y el destino de los humanos, solo que ahora en modo posmoderno. O sea, un mundo donde poderes seculares, explicaciones científico-técnicas, mercados globales y una creciente racionalidad instrumental terminan por desplazar a la universidad propiciada por la razón kantiana (Custer, 2014), igual que sucede con la torre de marfil de Humboldt (Reichenbach, 2013).

\section{CONCLUSIÓN MIRANDO AL FUTURO}

La pregunta que recorre los claustros ahora es esta: una vez que se complete la hegemonía de las Ingenierías y la Medicina, ¿cuáles facultades se harán cargo de llevar la antorcha?

Probablemente ninguna, dicen quienes se ocupan de imaginar escenarios futuros de la educación superior, partiendo del supuesto de que la universidad se halla a las puertas de innovaciones disruptivas (Blass \& Hayward, 2014; Barnett \& Bengtsen, 2017). Se provocarían, por el lado de la producción de conocimientos, reorganizaciones en la división del trabajo académico, impulsadas por procesos de convergencia tecnológicapresentes desde ya en la nueva economía-entre saberes, industrias, métodos y prácticas como la Nanotecnología, la Biotecnología, las Ciencias 
Cognitivas y las Ciencias Informáticas. Desde comienzos del presente siglo, la National Science Foundation (NSF) de los Estados Unidos se refiere a esta convergencia e integración de tecnologías bajo la sigla NIBC (nano-infobio-cognitive sciences) en áreas como Ciencia, Medicina, Electrónica, Medio Ambiente, Energía y Espacio (Bainbridge \& Roco, 2016). Estos fenómenos resultan difíciles de acomodar dentro de la milenaria estructura de las facultades inferiores y superiores, por lo que es probable que en el futuro los nuevos modos de producción de conocimiento se sitúen en las interfaces entre academia, laboratorios, empresas, redes y mercados (Nowotny, Scott \& Gibbons, 2001). Consecuencia de lo anterior, la antorcha que ilumina el camino habrá pasado entonces a nuevas constelaciones híbridas, dejando atrás la querella de las facultades y sus bases disciplinarias tradicionales.

Por el lado de la docencia, en tanto, podría suceder algo semejante: multiplicación de módulos y programas de todo tipo; uso de diversas plataformas tecnológicas y métodos de enseñanza y aprendizaje; ilimitadas oportunidades para que las personas definan sus trayectorias formativas; articulación flexible de instancias docentes basadas en el uso del continuo práctica-reflexión-teoría en ambas direcciones; utilización de formas variadas de evaluación, reconocimiento y certificación de estas experiencias de aprendizaje; y diferentes espacios de ocurrencia de estos procesos en la escala completa desde lo local a lo global y sus puntos intermedios (Peters, 2010).

En cualquier caso, las organizaciones académicas del futuro deberán acostumbrarse a la diversidad como principio básico. Enfrentadas a esta situación, referentes normativos tradicionales, como Bildung, perderían vigencia y dejarían de ser plausibles, según hemos examinado en este artículo. En este contexto no puede sorprender que las discusiones filosóficas contemporáneas acerca de los propósitos de la universidad partan de la ausencia de un ideal comprehensivo para estas instituciones y enfaticen, en cambio, la importancia de su capacidad reflexiva y de poner en cuestión el mismo conocimiento que generan y transmiten (Barnett, 2000; Derrida, 2002).

Por el contrario, reflexiones que no consideren el carácter socialmente construido de la institución — por ejemplo, la visión de la universidad como bien público, o como agente de crítica, o como empresa, entre otras-quedan sujetas ahora a un escrutinio ideológico. Siguiendo a Kant (1798/2004), la razón tiene el «derecho de hablar con franqueza» (p. 23) no solo de la sociedad sino, además, sobre los propios presupuestos de su enunciación. La modernidad de la universidad se expresa hoy, por tanto, de manera paradójica como la puesta en cuestión de la propia institución. 


\section{NOTAS}

1 Artículo elaborado en el contexto del Proyecto FONDECYT N. ${ }^{\circ}$ 1180746-Sistema universitario chileno: gobernanza del capitalismo académico y calidad de las instituciones. Agradecemos los comentarios de los evaluadores. Cualquier error u omisión es responsabilidad de los autores.

2 La distinción entre Facultades superiores y una inferior, tiene que ver con el carácter propedéutico que poseían los estudios impartidos en esta última, la Facultad de artes que antiguamente enseñaba el trívium y el quadrivium y que, más adelante, pasó a llamarse de filosofía. El orden jerárquico entre las ramas del saber tiene un importante valor de conocimiento, organizacional, de poder y ritual (Füssel, 2011). Desde Kant, particularmente en la tradición de la cultura alemana, la primacía intelectual y formativa se atribuye a la Facultad de filosofía, mientras las Facultades profesionales son consideradas de menor valía por su carácter vocacional y utilitario (Röhrs, 1996; Kantasalmi, 2015). Entre los académicos de humanidades y ciencias naturales florece una ideología que opone una formación (Bildung) basada en el intelecto (Geist) sin otro propósito que el autocultivo, frente a la enseñanza utilitaria de las Facultades profesionales que, despectivamente, aquellos otros califican de «estudio de ganapanes» (Brotstudium) (Turner, 1971, p. 152).

3 Sobre la recepción de esta idea en América Latina, ver Bernasconi (2008). Para el caso de España, véase Bermejo Castrillo (2008).

4 Sobre este concepto, ver Hamann (2015).

5 A lo largo de los cuatro volúmenes de la historia de las universidades europeas, los que cubren desde el siglo XII hasta comienzos del siglo XXI, puede observarse cómo disminuye progresivamente la importancia atribuida a la Facultad de filosofía, al punto que, en el último de aquellos volúmenes, que incluye desde la segunda posguerra hasta el presente, prácticamente no se la menciona y no ocupa un lugar propio entre las principales Facultades (Rüegg, 2011).

6 Es interesante observar, como señala Kant en un famoso pasaje, que la propia universidad moderna adoptó-a través de sus Facultades-una forma de división del trabajo que remeda a la organización industrial, orientada en este caso hacia la producción de conocimiento (Kant, 1798/2004, p. 19).

7 Traducción de los autores del alemán.

8 Las citas a Weber (1917/2008) provienen de la conferencia sobre la ciencia como profesión dictada el 7 de noviembre de 1917 en la Universidad de Múnich, según el texto y páginas de la edición de Dreijmanis que aparece en las referencias. Para los textos en castellano se utilizó la traducción de Abellán (Weber, 1917/2013).

9 La traducción usual de esta frase al castellano es más simple y directa: "Creo porque es absurdo». Sobre su trayectoria discursiva y la veracidad de su atribución primero a San Agustín y más comúnmente a Tertuliano y su difusión dentro de la teología cristiana, véase Harrison (2017, p. 361). 


\section{REFERENCIAS BIBLIOGRÁFICAS}

Ash, M.G. (2006). Bachelor of what, master of whom? The Humboldt myth and historical transformations of higher education in German-speaking Europe and the US. European Journal of Education, 41(2), 245-67. doi: j.1465-3435.2006.00258.x

Ash, M. G. (2008). From 'Humboldt' to 'Bologna': History as discourse in higher education reform debates in German-speaking Europe. In B. Jessop, N. Fairclough, \& R. Wodak (Eds.), Education and the knowledgebased economy in Europe (pp. 4162). Rotterdam, Netherland: Sense Publishers.

Abellán, J. (2008). La idea de Universidad de Wilhelm von Humboldt. En F. Oncina Coves (Ed.), Filosofía para la universidad, Filosofía contra la universidad (De Kant a Nietzsche) (pp. 273-296). Madrid: Editorial Dykinson.

Bainbridge, W.S. \& Roco, M.C. (2016). Science and technology convergence: With emphasis for nanotechnologyinspired convergence. Journal of Nanoparticle Research, 18(7), 211-230. doi: 10.1007/s11051- 016-3520- 0

Barkhoff, J. (2017). Romantic sociability, aesthetics and politics. En A.J. Webber (Ed.), Cambridge companion to the literature of Berlin (pp. 33-51). Cambridge, UK: Cambridge University Press.

Barnett, R. (2000). Realizing the university in an age of supercomplexity. Buckingham, UK: The Society for Research into Higher Education \& Open University Press.

Barnett, R., \& Bengtsen, S. (2017). Universities and epistemology: From a dissolution of knowledge to the emergence of a new thinking. Education Sciences, 7(1), 38-50. doi: 10.3390/educsci7010038

Becker, G.S. (1994). Human capital: A theoretical and empirical analysis with special reference to education. Chicago, USA: The University of Chicago Press.

Benziman, G. (2018). Goethe's Wilhelm Meister and the refusal to grow up: The dialectics of Bildung. Goethe Yearbook, North American Goethe Society, 25, 217-237. doi: gyr.2018.0011

Bermejo Castrillo, M.A. (2008). La universidad europea entre Ilustración y liberalismo. Eclosión y difusión del modelo alemán y evolución de otros sistemas nacionales. En F. Oncina Coves (Ed.), Filosofía para la universidad, Filosofía contra la universidad (De Kant a Nietzsche) (pp. 49-165). Madrid: Editorial Dykinson.

Bernasconi, A. (2008). Is there a Latin American model of the university? Comparative Education Review, 52(1), 27-52. doi: ihe.2008.52.8031

Blass, E. \& Hayward, P. (2014). Innovation in higher education: Will there be a role for "the academe/ university" in 2025? European Journal of Futures Research, 2(41). Doi 10.1007/s40309-014-0041-X

Bleiklie, I., Enders, J., \& Lepori, B. (2015). Organizations as penetrated hierarchies: Environmental pressures and control in professional organizations. Organization Studies, 36(7), 873-896.

Brandt, R. (2008). La contienda de las Facultades. Determinación racional y determinación ajena en la universidad kantiana. En F. Oncina Coves (Ed.), 
Filosofía para la universidad, Filosofía contra la universidad (De Kant a Nietzsche) (pp. 167-197). Madrid: Editorial Dykinson.

Brunner, J. J. (2017). La economía política del capitalismo académico: El caso de Chile. En H. Lavados y A.L. Durán (Eds.), Desafíos para el desarrollo universitario en Chile (pp. 159-231). Valencia: Tirant Humanidades.

Cantwell, B. \& Kauppinen, I. (Eds.) (2014). Academic capitalism in the age of globalization. Baltimore, MD, USA: Johns Hopkins Press.

Clark, B.R. (1998). Creating entrepreneurial universities: Organizational pathways of transformation. Oxford, UK: Pergamon-Elsevier.

Clark, C. (2006). Iron kingdom. The rise and downfall of Prussia, 1600-1947. Cambridge, MA., USA: The Belknap Press of Harvard University Press.

Custer, O. (2014). A certain truth: Derrida's transformation of the Kantian heritage. En Z. Direk \& L. Lowler (Eds.), A Companion to Derrida (pp. 45-56). Chichester, UK: Wiley Blackwell.

Derrida, J. (2002). La universidad sin condición. Madrid: Editorial Trotta.

Etzkowitz, H. (2017). The entrepreneurial university. In J.C. Shin \& P. Teixeira (Eds.), Encyclopedia of international higher education systems and institutions. Dordrecht, Netherland: Springer Science+Business Media. doi: 10.1007/978-94-017-9553-1_17-1

Frijhoff. W. (2003a). Graduation and careers. In W. Rüegg (Ed.), A history of the university in Europe (Volume II, pp. 344-415). Cambridge, UK: Cambridge University Press.
Frijhoff, W. (2003b). Patterns. In W. Rüegg (Ed.), A history of the university in Europe (Volume II, pp. 43-110). Cambridge, UK: Cambridge University Press.

Füssel, M. (2011). The conflict of the faculties: Hierarchies, values and social practices in early modern German universities. In M. Feingold (Ed.), History of universities (Volume 25, pp. 80-110). Oxford, UK: Oxford University Press.

Gieysztor, A. (1992). Management and resources. In W. Rüegg (Ed.), A history of the university in Europe (Vol I, pp. 108-143). Cambridge, UK: Cambridge University Press.

Goethe, J.W. v. (1981). Werke: Hamburger Ausgabe in 14 Bänden. München, Deutschland: Deutscher Taschenbuch Verlag. \{Texto original publicado en 1832.\}

Habermas, J. (1987). The Idea of the university - Learning processes. New German Critique, 41, 3-22.

Hamann, J. (2011). «Bildung» in German human sciences: The discursive transformation of a concept. History of the Human Sciences, 24(5), 48-72. doi: $10.1177 / 0952695111421865$

Hamann, J. (2015). Die Geisteswissenschaften und ihr Bildungsdiskurs. Zur Kartierung eines vernachlässigten Gebiets der Wissenschaftssoziologie. Zeitschrift für Soziologie, 44(3), 180-196.

Harrison, P. (2017). «I believe because it is absurd»: The Enlightenment invention of Tertullian's credo. Church History, 86(2), 339-364. doi: S0009640717000531

Hastedt, H. (2012). Einleitung. In H. Hastedt (Ed.), Was ist Bildung?: 
JOSÉ JOAQUÍN BRUNNER, JULIO ROBERTO LABRAÑA VARGAS, FRANCISCO GANGA, EMILIO RODRÍGUEZ-PONCE

IDEA MODERNA DE UNIVERSIDAD: DE LA TORRE DE MARFIL AL CAPITALISMO ACADÉMICO

Eine Textanthologie (pp. 7-28). Stuttgart, Deutschland: Reclam.

Horlacher, R. (2014). ¿Qué es Bildung? $\mathrm{El}$ eterno atractivo de un concepto difuso en la teoría de la educación alemana. Pensamiento educativo. Revista de Investigación Educacional Latinoamericana, 1(51), 35-45.

Howard, T. A. (2006). Protestant theology and the making of the modern German university. Oxford, UK: Oxford University Press.

Hütter, R. (2013). La universidad contemporánea ante la profética provocación de J.H. Newman. Humanitas, Revista de Antropología y Cultura Cristianas, 72, 752-779.

Jaeger, W. (1980). Paideia: Los Ideales de la Cultura Griega. México DF, México: Fondo de Cultura Económica.

Jessop, B. (2017). Varieties of academic capitalism and entrepreneurial universities. Higher Education, 73, 853-870. doi: 10.1007/s10734-0170120-6

Kant, I. (2004). El conflicto de las facultades. Buenos Aires, Argentina: Editorial Losada. [Texto original publicado en 1798]

Kant, I. (2013). ¿Qué es la Ilustración? Edición de R. A. Aramayo. Madrid: Alianza Editorial.

Kantasalmi, K. (2015). University and the contingencies in politics of research, development and innovations: Finnish reform of doctoral schools and doctoral programs. In I. Langemeyer, M. Fischer, \& M. Pfadenhauer (Eds.), Epistemic and learning cultures: wohin sich Universitäten entwickeln (pp. 115137). Weinheim, Bergstr, Germany: Beltz Juventa.
Krücken, G. \& Meier, F. (2006). Turning the university into an organizational actor. In G.S. Drori, J.W. Meyer, \& H. Hwang (Eds.), Globalization and organization: World society and organizational change (pp. 241-257). Oxford, UK: Oxford University.

Llinares, J.B. (2008). Buscando espacios para la verdad: Nietzsche y la filosofía en la universidad. En F. Oncina Coves (Ed.), Filosofía para la universidad, Filosofía contra la universidad (De Kant a Nietzsche) (pp. 311-360). Madrid: Editorial Dykinson.

Lyotard, J.F. (1984). The postmodern condition: A report on knowledge. Translated by Brian Massumi. Minneapolis, USA: University of Minnesota Press.

McClelland, C. E. (2008). State, society, and university in Germany, 1700-1914. Cambridge, UK: Cambridge University Press.

Medina Echavarría, J. (1967). Filosofía, educación y desarrollo. México D.F., México: Siglo XXI Editores.

Nordenbo, S.E. (2003). Bildung and the thinking of Bildung. In L. Lovlie, K.P. Mortensen \& S.E. Nordenbo (Eds.), Educating humanity. Bildung in postmodernity (pp. 15-36). Cornwall, UK: Blackwell Publishing.

Nowotny, H., Scott, P. \& Gibbons, M. (2001). Re-thinking science: Knowledge and the public in an age of uncertainty. Cambridge, UK: Polity Press.

Nybom, T. (2007). A rule-governed community of scholars: The Humboldt vision in the history of the European university. En P. Maassen \& J.P. Olson (Eds.), University dynamics and European integration (pp. 55-79). Dordrecht, Netherland: Springer. 
Paradeise, C., Reale, E., Bleiklie, I., \& Ferlie, E. (Eds.). (2009). University governance. Dordrecht, Netherland: Springer.

Peters, M.A. (2010). Re-Imagining the University in the Global Era. Policy Futures in Education, 8(2), 151-165. doi: 10.2304/pfie.2010.8.2.151

Pinheiro, R. (2015). Humboldt meets Schumpeter? Interpreting the 'Entrepreneurial turn' in European higher education. ISL Working Paper, 2.

Readings, B. (1996). The university in ruins. Cambridge, Mass., USA: Harvard University.

Reichenbach R. (2013). Freiheit und Einsamkeit in der Massenuniversität. In N. Ricken, H.C. Koller \& E. Keiner (Eds.), Die Idee der Universität revisited (pp. 79-100). Wiesbaden, Deutschland: Springer VS.

Ringer, F. (1990). The decline of the German mandarins. The German academic community, 1890-1933. Hannover, Germany: Wesleyan University Press.

Ringer, F. (2004). Max Weber. An intellectual biography. Chicago, USA: The University of Chicago Press.

Röhrs, H. (1996). The classical German concept of the university and its influence on higher education in the United States. Frankfurt am Main, Germany: Peter Lang.

Rothblatt, S. (2011). Curriculum, students, education. En W. Rüegg (Ed.), A history of the university in Europe (Vol. IV, pp. 238-275). Cambridge, UK: Cambridge University Press.

Rüegg, W. (Ed.) (2004). A history of the university in Europe. Volume 3:
Universities in the Nineteenth and Early Twentieth Centuries (1800-1945). Cambridge, UK: Cambridge University Press.

Rüegg, W. (2011). Themes. En W. Rüegg (Ed.), A history of the university in Europe. Vol. 4: Universities Since 1945. Cambridge, UK: Cambridge University Press.

Schwerhoff, G. (2013) Early modern violence and the honour code: From social integration to social distinction? Crime, Histoire et Sociétés, 17(2), 27-46. doi: 10.1080/13507486.2014.949631

Shapin, S. (2012). The ivory tower: The history of a figure of speech and its cultural uses. BJHS, 45(1), 1-27. doi: $10.1017 / \mathrm{S} 0007087412000118$

Slaughter, S. (2014). Retheorizing academic capitalism: Actors, mechanisms, fields, and networks. In B. Cantwell \& I. Kauppinen (Eds.), Academic capitalism in the age of globalization (pp. 20-32). Baltimore, MD, USA: Johns Hopkins Press.

Snow, C.P. (2012). The two cultures. With Introduction by $S$. Collini. Cambridge, UK: Cambridge University Press.

Turner, R. S. (1971). The growth of professorial research in Prussia, 1818 to 1848 . Causes and context. Historical Studies in the Physical Sciences, 3, 137182. doi: $10.2307 / 27757317$

Verger, J. (1992). Patterns. En W. Rüegg (Ed.), A history of the university in Europe (Vol I, pp. 35-74). Cambridge, UK: Cambridge University Press.

Weber, M. (2008). Max Weber's complete writings on academic and political vocations. Edited and with an Introduction by J. Dreijmanis. Translation by G. C. Wells. New York, USA: Algora Publishing. [Texto original publicado en 1917] 
Weber, M. (2013). La ciencia como profesión. Edición de Joaquín Abellán. Madrid: Grupo Editorial Siglo Veintiuno. [Texto original publicado en 1917]

Weber, M. (2014). Economía y sociedad. Nueva edición, revisada, comentada y anotada por F. Gil Villegas. México, DF,
México: Fondo de Cultura Económicas. [Texto original publicado en 1922]

Zovko, M.E. \& Dillon, J. (2018). Humanism vs. competency: Traditional and contemporary models of education, Educational Philosophy and Theory, 50(6-7), 554-564, doi: $10.1080 / 00131857.2017 .1375757$ 


\section{PERFIL ACADÉMICO Y PROFESIONAL DE LOS AUTORES}

José Joaquín Brunner. Sociólogo, PhD por la Universidad de Leiden, Países Bajos, Profesor Titular de la Universidad Diego Portales de Chile donde dirige la Cátedra UNESCO de Políticas Comparadas de Educación Superior. Sus líneas de investigación son sociología comparada de políticas y sistemas de educación superior, economía política y gobernanza de sistemas nacionales de educación superior, y análisis organizacional de mercados de la educación superior.

Julio Labraña. Sociólogo, Magíster en Análisis Sistémico aplicado a la Sociedad, PhD por la Universidad Witten/Herdecke, Alemania. Investigador asociado del Centro de Políticas Comparadas de Educación de la Universidad Diego Portales. Sus líneas de investigación incluyen sociología de la educación superior, teoría de sistemas sociales, y análisis de las relaciones entre cambios semánticos y estructurales.

Francisco Ganga. Administrador público, licenciado en administración, Magíster en Administración de Empresas, DEA en Organización de Empresas, Doctor en Administración de Empresas, Doctor en Gestión Estratégica y NI y Postdoctorado en Ciencias Humanas. Además es Profesor Titular del Departamento de Ciencias del Desarrollo e Investigador del Programa de Investigación en Gobernanza e Inclusión Organizacional de la Universidad de Los Lagos, Chile. Sus principales líneas de investigación incluyen administración y gobernanza universitaria.

Emilio Rodríguez-Ponce. Profesor Titular y Rector de la Universidad de Tarapacá. Doctor en Ciencias Económicas y Empresariales por la Universidad Complutense de Madrid y Doctor en Ciencias de la Educación por la Universidad Autónoma de Barcelona. Sus líneas de investigación consideran el campo de la educación superior, los procesos de toma de decisiones, la gestión del conocimiento y el liderazgo.

Dirección de los autores: Centro de Políticas Comparadas de Educación Universidad Diego Portales

Avenida Ejército, 260

Santiago, Chile

E-mail: josejoaquin.brunner@gmail.com Julio.LabranaVargas@uni-wh.de fganga@ulagos.cl erodriguez@uta.cl

Fecha Recepción del Artículo: 01. Septiembre. 2018 Fecha Modificación del Artículo: 12. Diciembre. 2018 Fecha Aceptación del Artículo: 16. Diciembre. 2018 Fecha Revisión para Publicación: 01. Febrero. 2019 